\title{
Fibroblast Growth Factor-2 Promotes Axon Branching of Cortical Neurons by Influencing Morphology and Behavior of the Primary Growth Cone
}

\author{
Györgyi Szebenyi, Erik W. Dent, John L. Callaway, Chad Seys, Helen Lueth, and Katherine Kalil \\ Department of Anatomy and Neuroscience Training Program, University of Wisconsin, Madison, Wisconsin 53706
}

Interstitial branching is an important mechanism for target innervation in the developing CNS. A previous study of cortical neurons in vitro showed that the terminal growth cone pauses and enlarges in regions from which interstitial axon branches later develop (Szebenyi et al., 1998). In the present study, we investigated how target-derived signals affect the morphology and behaviors of growth cones leading to development of axon branches. We used bath and local application of a targetderived growth factor, FGF-2, on embryonic pyramidal neurons from the sensorimotor cortex and used time-lapse digital imaging to monitor effects of FGF-2 on axon branching. Observations of developing neurons over periods of several days showed that bath-applied FGF-2 significantly increased growth cone size and slowed growth cone advance, leading to a threefold increase in axon branching. FGF-2 also had acute effects on growth cone morphology, promoting rapid growth of filopodia within minutes. Application of FGF-2-coated beads promoted local axon branching in close proximity to the beads. Branching was more likely to occur when the FGF-2 bead was on or near the growth cone, suggesting that distal regions of the axon are more responsive to FGF-2 than other regions of the axon shaft. Together, these results show that interstitial axon branches can be induced locally through the action of a targetderived growth factor that preferentially exerts effects on the growth cone. We suggest that, in target regions, growth factors such as FGF-2 and other branching factors may induce formation of collateral axon branches by enhancing the pausing and enlargement of primary growth cones that determine future branch points.

Key words: fibroblast growth factor; growth cone; collateral axon branching; cortical development; time-lapse imaging; cell culture
In many pathways, such as those projecting from the cerebral cortex, the primary growth cone at the axon tip guides the axon along the pathway but does not grow directly into targets. Instead, collateral branches extend interstitially from the axon shaft in the region of the target (O'Leary et al., 1990; Bastmeyer and O'Leary, 1996). For example, pathways arising from layer five projection neurons in the somatosensory cortex develop connections in vivo by branching of interstitial collaterals (O'Leary and Terashima, 1988; O'Leary et al., 1990; Norris and Kalil, 1992; Kuang and Kalil, 1994), and in vitro pyramidal neurons also develop interstitial axon branches (Szebenyi et al., 1998).

There is increasing evidence that target-derived cues affect not only the guidance of the primary axon (Letourneau, 1978; Gundersen and Barrett, 1979; McFarlane et al., 1995; Song et al., 1997) but also the extension of collateral branches. Recently, for example, the mammalian slit $2 \mathrm{~N}$ protein was shown to stimulate collateral axon branching on rat dorsal root ganglion neurons (Wang et al., 1999). Neurotrophins also regulate formation of terminal arbors and promote collateral sprouting. For example brain-derived neurotrophic factor (BDNF) augments the branching and complexity of optic axon terminal arbors in Xenopus

Received Nov. 20, 2000; revised Feb. 22, 2001; accepted March 13, 2001.

This work was supported by National Institutes of Health Grant NS 14428 to K.K. and National Institutes of Health Predoctoral Training Grant Award GM07507 to E.W.D. We thank Dr. Gerardo Morfini for helpful discussions.

Correspondence should be addressed to Dr. Katherine Kalil, University of Wisconsin, Department of Anatomy, 1300 University Avenue, Madison, WI 53706. E-mail: kakalil@facstaff.wisc.edu.

G. Szebenyi's present address: Department of Cell Biology, University of Texas Southwestern Medical Center, 5323 Harry Hines Boulevard, Dallas, TX 75390-9039. Copyright (C) 2001 Society for Neuroscience $0270-6474 / 01 / 213932-10 \$ 15.00 / 0$ tectum (Cohen-Cory and Fraser, 1995; Lom and Cohen-Cory, 1999), BDNF and neurotrophin-4/5 (NT-4/5) increase branch length on regenerating retinal ganglion cell axons (Sawai et al., 1996), NT-3 promotes collateral sprouting of corticospinal axons (Schnell et al., 1994; Grill et al., 1997), and fibroblast growth factors (FGFs) enhance neurite branching and process length of hippocampal neurons (Miyagawa et al., 1993; Aoyagi et al., 1994; Lowenstein and Arsenault, 1996; Shitaka et al., 1996).

In a previous study of cortical projection neurons in vitro (Szebenyi et al., 1998), we found that axon branches develop as a consequence of pausing behaviors by growth cones that enlarge and leave filopodial or lamellipodial remnants on the axon from which axon branches later emerge. Similar growth cone behaviors had been observed before axon branching in target regions of living cortical slices (Halloran and Kalil, 1994). Together, these results suggested that the growth cone could undergo pausing and branching in response to target-derived signals. Several in vitro studies have shown that local application of a growth factor can elicit lamellipodial activity (Ming et al., 1997) or branch-like protrusions (Gallo and Letourneau, 1998) along the axon shaft of cultured neurons. Therefore, in the present study, we used bath application of a widely distributed growth factor, FGF-2, to investigate how target-derived signals affect the morphology and behaviors of growth cones and development of axon branches. To determine whether some regions of the axon may be more sensitive to branch-inducing growth factors, we also applied FGF-2coated beads to localized regions of the axon shaft and growth cone. Time-lapse imaging was used to monitor the effects of FGF-2 on axon branching of cultured embryonic pyramidal neurons from the sensorimotor cortex. 


\section{MATERIALS AND METHODS}

Cell culture. All reagents were purchased from Life Technologies (Grand Island, NY) unless specified. Cultures were prepared from cortical tissue obtained from embryonic day 15 Syrian golden hamster fetuses $(\mathrm{Me}$ socricetus auratus). The pregnant mother was anesthetized with $\sim 30 \mathrm{mg}$ of Nembutal (Abbot Laboratories, North Chicago, IL). Fetuses were removed under aseptic conditions and transferred to cold PBS. Skulls were removed, and the forebrains were transferred to ice-cold dissection medium (Hibernate-E supplemented with B27, $0.3 \%$ glucose, $1 \mathrm{~mm}$ L-glutamine, and $10 \mu \mathrm{M}$ gentamycin sulfate). After the meninges were removed, the sensorimotor cortex was dissected out and minced into small pieces. Cortical pieces were dissociated with $0.025 \%$ trypsin for 15 min at $37^{\circ} \mathrm{C}$ in HBSS (without magnesium and calcium chloride), 0.27 mM EDTA, and $0.05 \%$ DNase I (Sigma, St. Louis, MO) with gentle agitation every $5 \mathrm{~min}$, followed by gentle trituration four to six times in serum-containing media (SCM) $[10 \%$ fetal bovine serum (FBS) $(\mathrm{Hy}-$ clone, Logan, UT), 1× B27 supplement, $0.3 \%$ glucose, $1 \mathrm{~mm}$ L-glutamine, and $10 \mu \mathrm{M}$ gentamycin sulfate in Neurobasal medium]. Undissociated pieces of tissue were allowed to settle, the cell suspension was collected, and the trituration was repeated twice with fresh SCM. Pooled cell suspensions were centrif uged at $8 \times g$ for $5 \mathrm{~min}$, resuspended in fresh SCM, and counted on a hemocytometer.

Neurons were cultured on plain (Fisher Scientific, Itasca, IL) or etched-grid (Bellco, Vineland, NJ) coverslips coated with $0.5 \mathrm{mg} / \mathrm{ml}$ poly-D-lysine (Sigma) in borate buffer for $1 \mathrm{hr}$, rinsed three times with distilled water, and then coated with $20 \mu \mathrm{g} / \mathrm{ml}$ laminin in Neurobasal medium at $36^{\circ} \mathrm{C}$ for at least $3 \mathrm{hr}$. For immunocytochemistry, neurons were grown on Lab-Tek eight-well chamber slides (Nunc, Naperville, IL) coated in a similar manner. Neurons were plated in SCM at densities ranging from 1000 to $2000 \mathrm{cells} / \mathrm{cm}^{2}\left(5000-10,000 \mathrm{cells} / \mathrm{cm}^{2}\right.$ for immunocytochemistry). After 1-2 hr, medium was changed to a serum-free formulation (SFM) (Neurobasal medium with B27 supplement, 0.3\% glucose, $1 \mathrm{~mm}$ L-glutamine, and $10 \mu \mathrm{M}$ gentamycin sulfate). Cultures were maintained at $36^{\circ} \mathrm{C}$ in $5 \% \mathrm{CO}_{2}$ for $4-6 \mathrm{~d}$; for some experiments, approximately half of the medium was exchanged with fresh SFM every $2 \mathrm{~d}$. These conditions resulted in neuronal cultures that remained viable for $5-7 \mathrm{~d}$ in the presence of very few glial cells $(<5 \%)$. For acute observations, experiments were performed on cells that were cultured for $24 \mathrm{hr}$.

For the bath application experiments, FGF-2 (Promega, Madison, WI) in SFM was added at various times to the dishes in a volume of $<5 \%$ of the total medium volume. For some experiments, heparin (from porcine intestinal mucosa; Sigma) was also added; it was first dissolved in PBS and boiled for $5 \mathrm{~min}$. These factors were washed out in some experiments with at least two complete changes of medium.

Heparin-FGF beads. Amino-coupled polystyrene microspheres $3 \mu \mathrm{m}$ in diameter were obtained by custom order from Molecular Probes (Eugene, OR). Two types of beads were used, either yellow-green fluorescent or nonfluorescent, so that both could be added to each dish, and their identity was determined using epifluorescence microscopy. Beads were covalently coupled to heparin. The high-affinity of this molecule for FGF-2 then allowed us to noncovalently couple the growth factor to the beads in its biologically active form (Niswander et al., 1993). Beads were washed two times in PBS and pelleted by centrifugation at $15,000 \times \mathrm{g}$ for $7 \mathrm{~min}$. Then the beads were resuspended in $8 \%$ glutaraldehyde in PBS and incubated for 4-6 hr (all incubations were performed at room temperature with end-over-end mixing of the tubes). After two washes in PBS, beads were incubated in $40 \mu \mathrm{g} / \mathrm{ml}$ heparin in PBS overnight. (Heparin was added from a $10 \mathrm{mg} / \mathrm{ml}$ heparin stock solution boiled for $5 \mathrm{~min}$.) Heparin-coated beads were pelleted and incubated in $0.2 \mathrm{M}$ ethanolamine in PBS (Polysciences, Warrington, PA) for $30 \mathrm{~min}$. Beads were washed twice in PBS and transferred to a new tube in PBS. Heparin-coupled beads were incubated in either $100 \mathrm{ng} / \mu \mathrm{l} \mathrm{FGF-2} \mathrm{in} \mathrm{PBS}$ or $100 \mathrm{ng} / \mu \mathrm{l}$ bovine serum albumin (Sigma) in PBS for $1 \mathrm{hr}$. Finally, beads were washed three times in PBS and resuspended in PBS. Beads were counted on a hemocytometer, and $15,000-20,000$ beads $/ \mathrm{cm}^{2}$ were applied to each dish $24 \mathrm{hr}$ after cell plating. Imaging began $2 \mathrm{hr}$ after bead addition, which was enough time for the majority of the beads to settle onto the substrate. Axons that were in contact with beads were imaged daily for $4-5 \mathrm{~d}$. Several experiments were performed so that each bead type (fluorescent or nonfluorescent) could be coupled to either FGF or BSA to control for any differences in the two types of beads. In some cases, heparin-FGF beads were stained with a rabbit polyclonal anti-FGF-2 antibody (Santa Cruz Biotechnology, Santa Cruz, CA) to assess whether the loading of beads with the growth factor was successful.

Microscopy and digital imaging. Imaging was performed as described previously (Szebenyi et al., 1998). Briefly, the culture dishes were sealed to prevent $\mathrm{pH}$ changes and transferred to the heated stage of a Zeiss (Thornwood, NY) 35M inverted microscope. Cortical neurons were viewed with a $20 \times / 0.5$ numerical aperture (NA) Neofluor objective under phase-contrast optics and imaged with a MicroMax cooled CCD digital camera (Princeton Instruments, Trenton, NJ) controlled by a Metamorph-based digital imaging system (Universal Imaging, West Chester, PA). To observe acute affects of FGF-2, we observed changes in growth cone morphology with differential interference contrast (DIC) optics and a $63 \times / 1.4$ NA Plan-Apochromat objective (Zeiss). For the bead experiments, a fluorescence image was acquired using a fluorescent filter set along with the phase image so that bead types could be distinguished.

For some experiments, neurons were selected and imaged before FGF treatment or bead application and were followed over the course of several days by using the etchings on the coverslips as landmarks. Other experiments involved imaging neurons at one time point only, $4 \mathrm{~d}$ after plating. In this case, unetched coverslips were used.

Immunocytochemistry. We stained for FGF receptors FR-1, FR-2, and FR-3 using rabbit polyclonal antibodies from Santa Cruz Biotechnology. Slides were fixed in $4 \%$ paraformaldehyde (Electron Microscopy Sciences, Fort Washington, PA) in Krebs' buffer (in mM: $145 \mathrm{NaCl}, 5 \mathrm{KCl}$, $1.2 \mathrm{CaCl}_{2}, 1.3 \mathrm{MgCl}_{2} \cdot \mathrm{H}_{2} 0,1.2 \mathrm{NaH}_{2} \mathrm{PO}_{4}, 10$ glucose, and $20 \mathrm{~mm}$ HEPES) with $0.4 \mathrm{M}$ sucrose (all reagents from Sigma) at $37^{\circ} \mathrm{C}$ for $20 \mathrm{~min}$. They were rinsed several times in PBS and then blocked-permeabilized in $10 \%$ FBS, $10 \%$ donkey serum (DS) (Jackson ImmunoResearch, West Grove, PA), and 0.05-0.1\% Triton X-100 (Sigma) in PBS at room temperature for $20 \mathrm{~min}$. Primary antibodies were diluted 1:100 in 3\% DS and $0.1 \%$ Tween 20 (Sigma) in PBS and incubated with cells at $4^{\circ} \mathrm{C}$ for $14-16 \mathrm{hr}$. Slides were washed four times for $8 \mathrm{~min}$ in $50-250 \mathrm{ml}$ of $0.2 \%$ Tween 20 in PBS. Cy3-conjugated anti-rabbit secondary antibody (Jackson ImmunoResearch) was applied in 3\% DS and $0.1 \%$ Tween 20 in PBS at a dilution of 1:200 for $1 \mathrm{hr}$ at room temperature. Slides were washed four times as before and coverslipped. They were observed using a rhodamine filter set with a $63 \times / 1.4$ NA Plan-Apochromat objective on a Zeiss $35 \mathrm{M}$ inverted microscope.

Heparin-FGF or heparin-BSA beads were stained with a rabbit polyclonal anti-FGF-2 antibody (Santa Cruz Biotechnology) at a dilution of 1:200. The procedure was identical to that used for the neurons, except that all incubations and washes were performed in microfuge tubes. The tubes were centrifuged between steps to pellet the beads so that the solutions could be removed.

Data analysis. All measurements were performed using the morphometric analysis tools of Metamorph. We chose for analysis neurons with pyramidal morphologies having only one process $100 \mu \mathrm{m}$ or longer after $4 \mathrm{~d}$ in culture. Such neurons were likely to be cortical projection neurons that branch interstitially in vivo (Kuang and Kalil, 1994) and in vitro (Szebenyi et al., 1998).

For the initial experiments on the effect of bath-applied FGF on branching, total axon length was measured, as well as the number of axon branches $>30 \mu \mathrm{m}$ in length. The number of primary axon branches was determined by counting branch points along the longest neurite. Additionally, we counted the number and position of branching regions and the number of branches per region. A single branching region was defined as a $70 \mu \mathrm{m}$ axon region that had one or several branches, the same definition as in our previous study (Szebenyi et al., 1998). For acute experiments (i.e., $1 \mathrm{hr}$ ), we chose neurons cultured for $24 \mathrm{hr}$ whose axons had not yet branched. For the bead experiments, regions of axon only 10 $\mu \mathrm{m}$ on either side of a bead directly touching the axon were considered. For these experiments, we narrowed the size of the branching region to ensure that only branches induced by the local release of FGF-2 were counted.

For the growth rate experiments, the time stamps associated with each image file by Metamorph were used to automatically calculate the time interval between successive images in Excel 97 (Microsoft, Redmond, WA). This time interval, combined with the change in axon length between the two images, allowed the calculation of average growth rate for that time interval. Some experiments involved measuring growth cone area; this was performed in Metamorph with the region tool.

Statistical analysis was performed using Excel 97 and Sigmaplot 4.0 (SPSS, Chicago, IL) softwares. Images were processed with Metamorph 3.6 and Photoshop 5.0 (Adobe Systems, Mountain View, CA). Figures 
A.

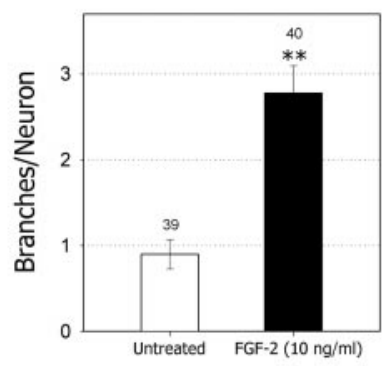

B.

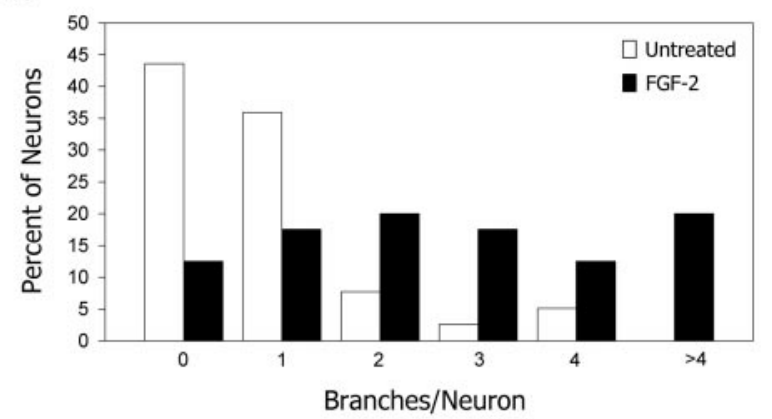

Figure 1. FGF-2 increases axon branching. $A$, Bar graph comparing branching of untreated versus FGF-2-treated neurons. Bars show mean number of axon branches per neuron. Both groups were also treated with $1 \mathrm{ng} / \mathrm{ml}$ heparin. Numbers above the bars indicate numbers of neurons in each group. Error bars are SEs. ${ }^{* *} p<0.001$ in a twotailed $t$ test. $B$, Frequency histogram showing the percentage of neurons with zero to more than four branches. Data plotted in $A$ and $B$ were from the same experiment. $C$, Examples of untreated (top) and FGF-2treated (bottom) neurons. Significant increase in branching occurred after treatment of the cortical neurons with FGF-2 after $24 \mathrm{hr}$ in culture. Images of neurons were obtained after $4 \mathrm{~d}$ in culture. In the treated neuron at the bottom left, growth cones on the axon branches were much larger than in the untreated control. In the treated neuron at the bottom right, the axon has branched into three major branches. On two of these, large numbers of developing branches are beginning to emerge form the lamellipodial expansions along the axon.

C.


were prepared directly from digital files. Images shown in the figures were modified using the unsharp mask filter and brightness-contrast adjustment tools in Photoshop to enhance detail and contrast.

\section{RESULTS}

\section{FGF-2 increases axon branching}

To assess the ability of various growth factors to induce branches on cortical axons, we treated cortical cultures with a battery of growth factors, including BDNF, NT-3, NT-4/5, ciliary neurotrophic factor (CTNF), glial-derived neurotrophic factor (GDNF), insulin-like growth factor 1 (IGF-1), and several members of the FGF family. Each of these peptide growth factors was bath applied in concentrations varying from 0.1 to $100 \mathrm{ng} / \mathrm{ml}$ to cultures of embryonic sensorimotor cortical neurons. Two to $4 \mathrm{~d}$ after application of growth factors, we counted and measured axon branches on living neurons with large pyramidal morphologies using computer-assisted morphometric imaging techniques. This survey revealed that, of all the growth factors tested, FGF-1, FGF-2, and IGF-1 were most effective in promoting branching on cortical axons. In contrast, NT-3 and CNTF treatment had only modest effects and BDNF, and NT-4/5 and GDNF had no effect on branching.

Based on this survey, we focused on the branching effects of FGF-2. Using concentrations from 0.1 to $100 \mathrm{ng} / \mathrm{ml}$, we found that $10 \mathrm{ng} / \mathrm{ml}$ elicited the most axon branches. Because it is known that heparin sulfate proteoglycans (HSPG) are required for many of the biological effects of FGF-2 (Guimond and Turnbull, 1999), we tested whether heparin, an HSPG substitute, augments FGF2 -induced branching. We found that $1 \mathrm{ng} / \mathrm{ml}$ heparin in combination with $10 \mathrm{ng} / \mathrm{ml}$ FGF-2 produced maximal branching effects, and thus heparin was included in all of the FGF-2-treated cultures. Two to $24 \mathrm{hr}$ after plating, FGF-2 and heparin were applied to the cultures. In most cases, FGF-2 remained in the culture medium for the duration of the experiments, although we found that application of FGF-2 for as little as $2 \mathrm{hr}$ was sufficient to elicit maximal branching. As shown in Figure 1, $3 \mathrm{~d}$ after treatment with FGF-2, the number of branches per axon had increased approximately threefold compared with untreated controls (Fig. $1 A$ ). Similar results were obtained with 1-20 ng/ml FGF-2 in 10 independent experiments. Both neurons and glia secrete FGFs, and immunostaining showed that, in our cultures, both neurons and glia express FGF-2 (data not shown). It is likely that heparin also facilitates the effects of endogenous FGF-2. Approximately $70 \%$ of FGF-2 treated axons had more than two branches, and $20 \%$ of the axons had more than four branches. In contrast in the untreated cortical cultures, almost half of the axons had no branches, only $20 \%$ had more than two branches, and none had more than four branches (Fig. 1B). As in our previous study of cortical axon branching (Szebenyi et al., 1998), branches were included only if they were at least $30 \mu \mathrm{m}$ long. Branches typically occurred in clusters, were often tipped by growth cones, and 
frequently rebranched (Fig. 1C). However, because we only counted primary branches, the threefold increase in branching is actually an underestimate of the total branching.

\section{FGF-2 treatment enlarges growth cones and slows their advance}

An important question is how FGF-2 increases axon branching. In a previous study, we found that the primary growth cones at the tips of cortical axons paused for extended time periods, increased fivefold in area, and left behind active regions along the axons from which branches subsequently emerged (Szebenyi et al., 1998). We also found that the larger the growth cone the more branches developed. Thus, one possibility is that FGF-2 increases axon branching by affecting the size and behaviors of the growth cone. To address this possibility, we first compared rates of growth cone extension in control versus FGF-2-treated cultures. As shown in Figure $2 A$, within $6 \mathrm{hr}$ after treatment with FGF-2, rates of growth cone extension had declined to approximately half of that of untreated controls. After $20 \mathrm{hr}$ of FGF-2 treatment, axonal growth rates were still slower compared with controls. Measurements of primary growth cones (at the axon tip), including the entire area of the lamellipodium, showed a small but significant increase after 2 hr of FGF-2 treatment (Fig. 2B). By $20 \mathrm{hr}$, growth cones had doubled in size compared with their size at $6 \mathrm{hr}$ and were nearly twice as large as untreated controls. Typically, the distal segment of the axon became spread and showed high filopodial activity (Fig. $2 C$ ). As shown in Figure $1 C$ (bottom left), even growth cones at the tips of primary and secondary branches were also noticeably enlarged compared with growth cones on branches of untreated neurons. This figure (bottom right) also shows a dramatic example of several enlarged remnants of growth cone lamellipodia on the shaft of an FGF-2-treated cortical axon. Numerous axon branches are beginning to emerge from these active regions. These results suggest that FGF-2 may increase axon branching by slowing and enlarging the growth cone.

\section{FGF-2 has acute effects on growth cone morphology}

Bath application of FGF-2 to the culture medium promoted branching over several days. To determine whether FGF-2 also has acute effects on cortical growth cones and axons, we performed time-lapse imaging of neurons for $20 \mathrm{~min}$ before adding FGF-2 (100 ng/ml) to the medium and for $40 \mathrm{~min}$ in the presence of FGF-2. Images were acquired every $30 \mathrm{sec}$. After addition of FGF-2, the most striking change at the growth cone was an increase in length of filopodia. For each growth cone $(n=4), \sim 20$ filopodia were measured from the edge of the lamellipodia to the filopodial tips at $3 \mathrm{~min}$ intervals during the entire $60 \mathrm{~min}$ observation period. Average filopodial length did not change significantly before addition of FGF-2. However, as shown in the graphs in Figure $3 A$, within $10 \mathrm{~min}$ of FGF-2 addition to the medium, filopodia that were typically $1-2 \mu \mathrm{m}$ had increased noticeably in length and appeared to be more active. By the end of the $40 \mathrm{~min}$, filopodia had at least doubled in length and in some cases grew to 5-6 $\mu \mathrm{m}$. Growth cones remained highly motile during the entire hour but did not advance. FGF-2 had no consistent effect on the size of the lamellipodia. In some cases after addition of FGF-2, we also observed new activity along the axon shaft as either filopodial or lamellar protrusions. In comparison, in control cultures treated with BSA $(n=3)$, growth cone filopodia remained relatively constant in length during the entire $60 \mathrm{~min}$ but were highly motile (Fig. $3 B$ ). Although we plotted filopodial length for individual growth cones (Fig. $3 A, B$ ), averages across all experi-
A.



B.

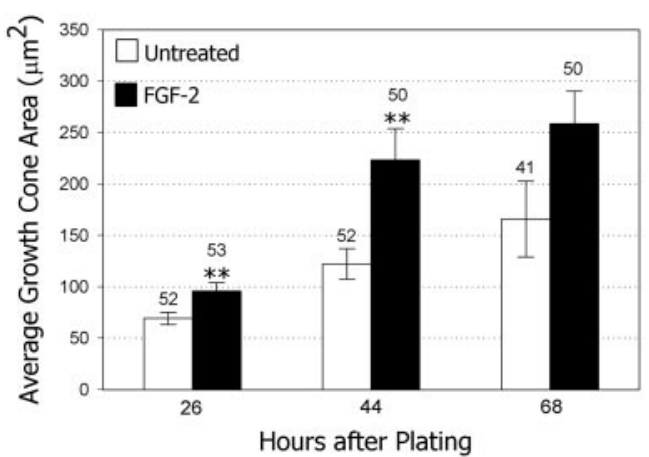

C.
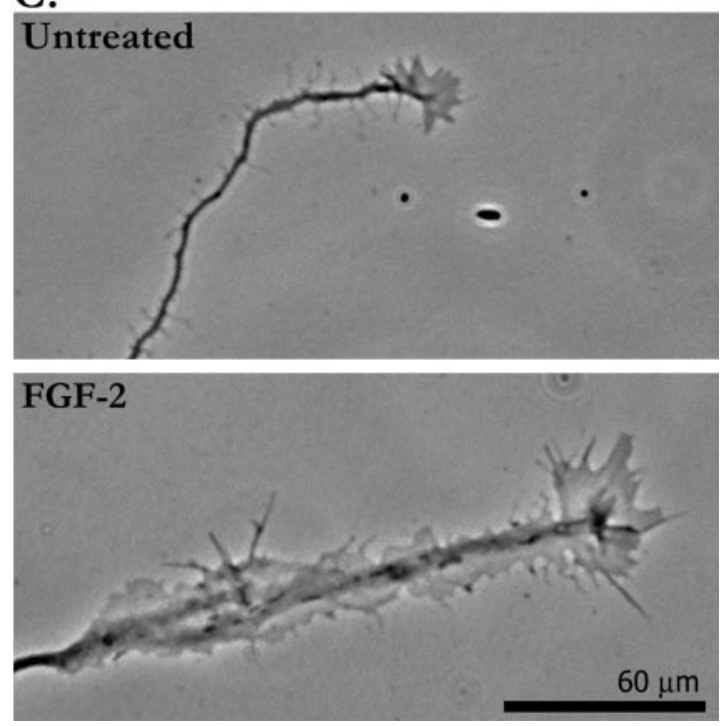

Figure 2. FGF-2 decreases rates of axon outgrowth and increases growth cone size. $A$, Bar graph comparing rates of axon outgrowth in untreated versus FGF-2-treated neurons. Both groups were also treated with $1 \mathrm{ng} / \mathrm{ml}$ heparin. FGF-2 treatment started at $24 \mathrm{hr}$ after cell plating. Cells were imaged at $3 \mathrm{hr}$ intervals to obtain growth rates. Numbers below the graph indicate the end of a $3 \mathrm{hr}$ time interval. The same population was followed over time, although some neurons did not survive until the end of the experiment. Numbers above the graphs refer to neurons measured at each time point. ${ }^{*} p<0.05$ in a two-tailed $t$ test. $B$, Bar graph comparing average growth cone size (area) of untreated versus FGF-2-treated neurons at various time points after plating. FGF-2 was added at $24 \mathrm{hr}$ after plating. Numbers refer to growth cones measured in the same population of neurons over time. Error bars are SEs in each of the graphs. ${ }^{*} p<0.01$ in a two-tailed $t$ test. $C$, Examples of untreated (top) and FGF-2-treated (bottom) growth cones showing increased growth cone size $2 \mathrm{~d}$ after FGF-2 treatment. 
Figure 3. FGF-2 increases filopodial length acutely on the growth cone and the axon shaft. $A$, Graph showing average length of growth cone filopodia before and after addition of FGF-2 to the medium. Points on the graph, with SE bars, show average length of all filopodia $(\sim 30)$ on a single growth cone (shown below) plotted at 3 min intervals. Representative images of the growth cone in DIC acquired before (left) and after (right) FGF-2 treatment were chosen from a $1 \mathrm{hr}$ time-lapse sequence. Times on images correspond to those shown in the graph. $B$, Graph showing average length of growth cone filopodia before and after addition of BSA to the medium as a control. Points on the graph, with SE bars, show average length of all filopodia $(\sim 20)$ on a single growth cone (shown below) plotted at 3 min intervals. Representative images of the growth cone in DIC acquired before (left) and after (right) BSA treatment were chosen from a 1 $\mathrm{hr}$ time-lapse sequence. Times on images correspond to those shown in the graph. $C$, Sequence of time-lapse images of an axon before $(-4$ and $-2 \mathrm{~min})$ and after $(20-50 \mathrm{~min})$ addition of FGF-2 to the medium. FGF-2 promotes growth and branching of the filopodium.


ments showed that filopodia doubled in length after application of FGF-2 (97.5 $\pm 16.5 \%$ increase) versus an increase of $17.3 \pm$ $13.5 \%$ after application of BSA. To assess FGF-2-induced filopodial activity along the axon shaft $(n=8)$, we determined the number and duration of filopodia $30 \mathrm{~min}$ before and $30 \mathrm{~min}$ after the addition of FGF-2. Of eight axons, six showed filopodial activity throughout the observation period. In contrast to filopodia on growth cones, axonal filopodia did not lengthen significantly in response to FGF-2, nor did FGF-2 promote filopodia de novo along the axon shaft. However, the duration of filopodia increased from $101 \pm 19 \sec (n=21)$ to $170 \pm 47 \sec (n=24)$. Filopodia extended transiently from the axon and within a few minutes could reach several micrometers in length and were even capable of branching before retracting (Fig. 3C). We do not know whether any of the filopodia on axons eventually progressed to become actual branches. Lamellipodia were also transient and traveled along the axon shaft in a wave-like manner. These results suggest that within minutes FGF-2 promotes morphological changes that precede axon branching.

\section{FGF receptors are present on embryonic cortical neurons}

To determine whether FGF receptors are expressed on cultured embryonic cortical neurons and if so to assess their distribution, we stained the cultures with antibodies against FGF receptors. Four tyrosine kinase FGF receptors have been identified (Szebenyi and Fallon, 1999). Each of these can bind to FGF-2 (Ornitz et al., 1996), but only FGF receptors 1-3 have been found in differentiated neurons (Itoh et al., 1994; Belluardo et al., 1997; Kalyani et al., 1999). Using antibodies against these three receptors, we found that FGF receptors 1 and 3 but not 2 were expressed on the cell body and processes of differentiated cortical neurons (Fig. 4). Immunostaining suggested that receptors were concentrated on large growth cones, but we did not attempt to quantitate these differences. These results suggest that FGF-2 has the potential to affect directly cortical neurons by binding to receptors broadly distributed over the neuron.

\section{FGF-2 acts locally to elicit axon branches}

Because FGFs are known to affect transcription rates of many genes (Szebenyi and Fallon, 1999), it is possible that FGF-2- induced branching results from a general enhancement of the basal metabolic rate of the neuron. If this were the case, then branches would be expected to arise randomly along the entire length of the axon. Another possibility is that FGF-2 produces its effects locally on certain regions of the axon, which would result in more branches in some regions of the axons than in others. To assess the distribution of FGF-2-induced branches along the axon, we compared the location of spontaneous branches in untreated cultures with those that developed after application of FGF-2. Branches in the untreated cultures were distributed uniformly along the axon (data not shown) but were rare in the distal fifth of the axon, in all likelihood because the interval between the development of this region of the axon and the time of observation was too short for branches to have developed. In contrast to untreated controls, cultures treated for $2 \mathrm{hr}$ with FGF-2 at 24 or $48 \mathrm{hr}$ after plating and then examined 2-3 d later had branches that were not uniformly distributed along the axons $(n=40$ branches per 43 axons at $24 \mathrm{hr} ; n=62$ branches per 58 axons at $48 \mathrm{hr}$ ) (Fig. 5). Instead, branches clustered in the region of the axon, which at the time of FGF-2 application comprised the distal half of the axon. Thus, although proximal regions of the axon were also exposed to FGF-2, branches were induced preferentially on the distal region of the axon.

Results thus far showed that FGF-2 has significant effects on the size and rate of extension of the terminal growth cone. To determine whether FGF-2 acts locally on the growth cone, we applied FGF-2-coated beads to the cultures and assessed whether beads in contact with growth cones promoted branching. Some of the beads contacted axon shafts, and we observed these regions as well for several days after bead application. Initially, we used FGF-2 covalently bound to polystyrene beads. However, these had no effects on axon branching (data not shown). Previous results showed that FGF-2 can be internalized and transported into the nucleus of neurons (Stachowiak et al., 1997; Mufson et al., 1999), and internalization is required for some of the biological effects of FGF-2 (Delrieu, 2000). Therefore, we loaded FGF-2 onto heparin-coated beads, according to established methods (Niswander et al., 1993). To avoid raising the overall concentrations of FGF-2 through diff usion of FGF-2 from the beads into the culture medium, the beads were applied at very low densities 

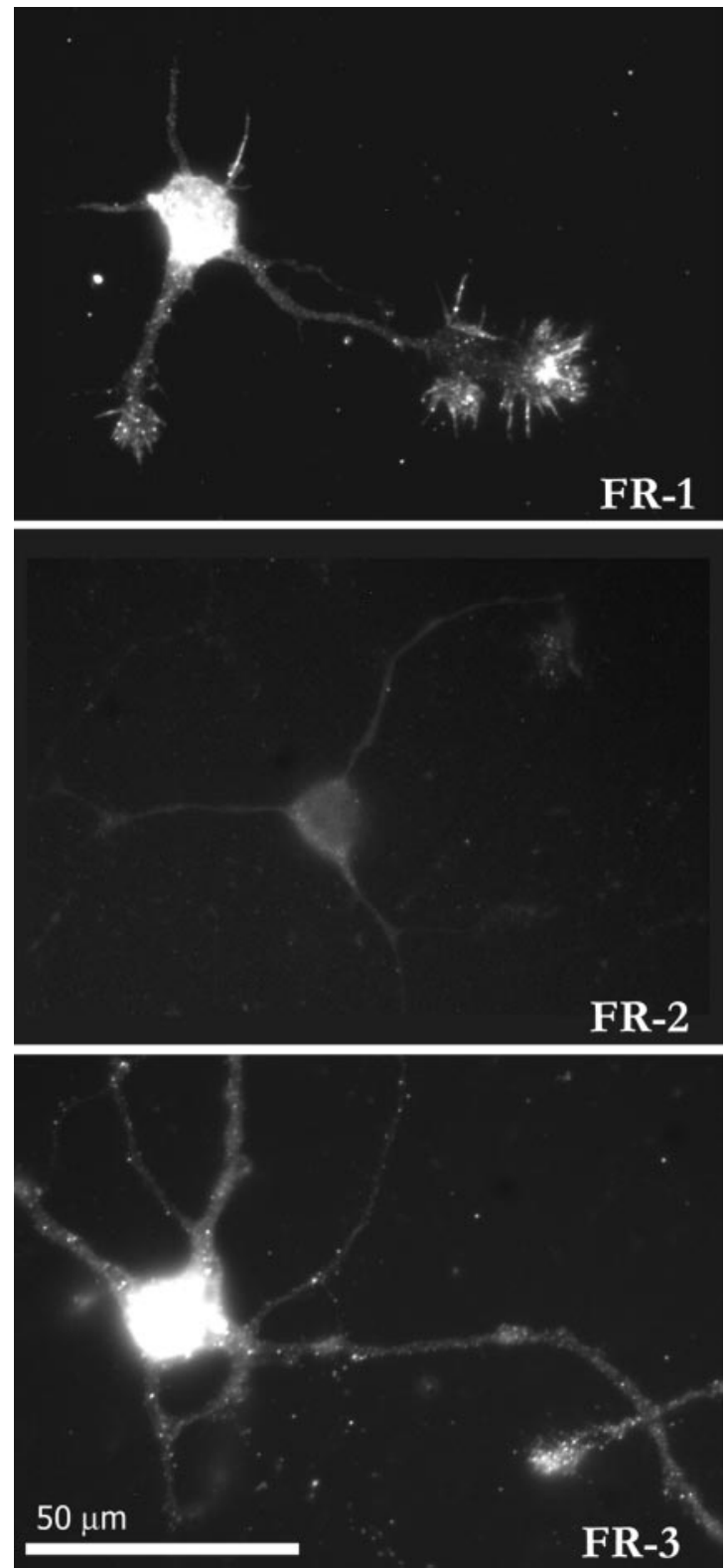

Figure 4. FGF receptors are expressed on cortical neurons. Examples of neurons stained immunocytochemically with antibodies to FGF receptors FR-1, FR-2, and FR-3. Both FR-1 and FR-3 but not FR-2 are expressed. FR-1 and FR-3 are seen throughout neurons but are concentrated on cell bodies and growth cones.

to the cultures. As a control, we applied heparin beads that had been soaked in BSA. Very few branches developed after contact with BSA-coated beads, whether or not FGF-2-coated beads were also added to the cultures. For BSA beads alone only, 6\% (1 of 17) were in close proximity to branches. Similarly, when FGF-2 beads were also present, only 4\% (1 of 25) of the BSA beads were near branches. Although we did not directly measure levels of FGF-2 in cultures with FGF-2-coated beads, these results show that the concentration of FGF-2 in the cultures did not produce an overall bath effect. Therefore, application of FGF-2-coated heparin beads is an appropriate strategy for studying local effects of FGF-2, although the low density of beads resulted in very few bead axon contacts such that a single axon was never contacted by more than one bead. Therefore, in the following data, the num-

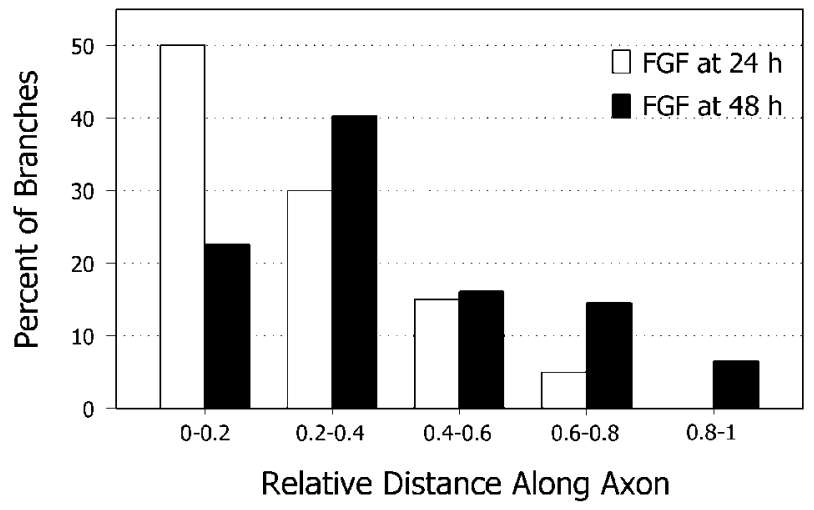

Figure 5. FGF-2 induces branches preferentially at distal regions of the axon. Bar graph compares positions of axon branches elicited with FGF-2 added at different stages of development. Spontaneously formed axon branches were scattered evenly along the axon (data not shown). Branches induced by bath application of FGF-2 tended to cluster at regions along the axon corresponding to positions of the primary growth cone at the time when FGF-2 was applied. Distances are given in relative distances from the cell body and do not denote actual axon lengths. The results graphed were obtained from one experiment $(n=40$ branches per 43 axons at $24 \mathrm{hr} ; n=62$ branches per 58 axons at $48 \mathrm{hr}$ ), but each experiment was performed three times with similar results. FGF-2 was applied for $2 \mathrm{hr}$. Branching was assessed at $96 \mathrm{hr}$ after plating.

bers of total beads are equivalent to the number of axons examined. Some beads contacted cell bodies, but the fates of these neurons were not monitored.

FGF-2-coated beads in contact with axons or growth cones increased the number of branches only within $10 \mu \mathrm{m}$ on either side of the bead (see Materials and Methods; Fig. 6). Along the rest of the axon, whether in contact with a BSA (Fig. 6A) or an FGF-2 bead (Fig. 6B), branches were randomly distributed. Of 12 branches that developed on axons contacted by BSA beads, only 1 branch (8\%) was within $10 \mu \mathrm{m}$ of the bead. In contrast, of 28 branches that developed on axons contacted by FGF-2-coated beads, $16(57 \%)$ were located within $10 \mu \mathrm{m}$ of the bead. These data show that FGF-2-coated beads act locally to promote axon branching. Therefore, all further analysis describes branches within $10 \mu \mathrm{m}$ of beads. FGF-2-coated beads were compared with BSA control beads in their ability to promote cortical axon branching (Fig. $6 C$ ). Of a total of 30 BSA beads in contact with an axon or a growth cone, only 1 bead (3\%) was located within 10 $\mu \mathrm{m}$ of a branch. In contrast, of a total of 44 FGF-2-coated beads that contacted an axon or a growth cone, 16 beads $(36 \%)$ elicited a branch within $10 \mu \mathrm{m}$ of a bead. These results show that FGF2-coated beads are highly effective in promoting growth of axon branches. In fact, the magnitude of the increase in branching $(\sim 12 \times)$ from a point source of FGF-2 beads versus control beads is much larger than the difference between bath application of the growth factor and untreated controls $(\sim 3 \times)$.

We followed the development of FGF-2-induced branches over $3 \mathrm{~d}$ starting from the time when the bead first contacted the axon or growth cone (Fig. 7). Branches formed within 24-72 hr after contact between a bead and the axon or growth cone. All of the branches were stable over the entire observation period, grew to lengths that averaged over $90 \mu \mathrm{m}$, and sometimes rebranched. Branches continued to elongate even when the bead had moved away from contact with the axon $(n=4)$.

Because bath application of FGF-2 suggested that branches were more likely to be induced on distal regions of the axon, we were interested in whether FGF-2-coated beads were also more 

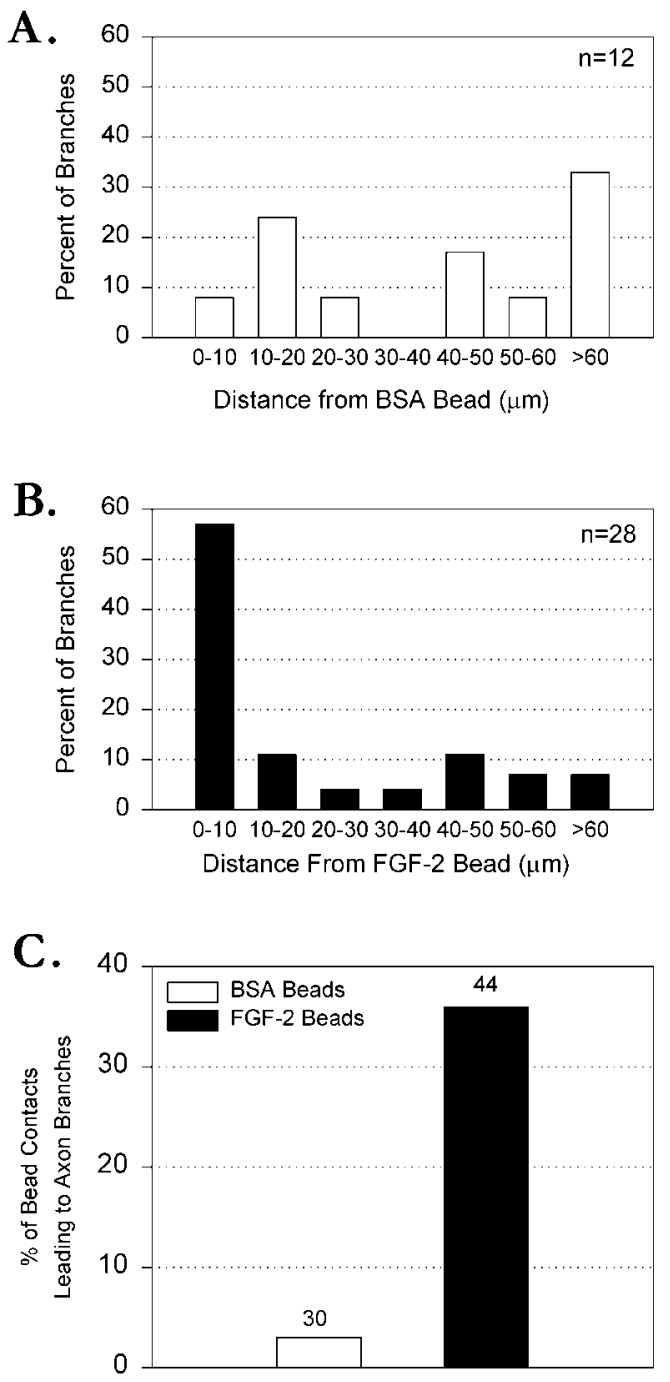

Figure 6. FGF-2-coated beads induce local axon branching. $A$, Bar graph showing the distribution of branches along axons contacted by BSA beads. $n$ refers to the number of axon branches found at any part of the axons contacted by beads. Note that branches are distributed randomly along the axons. $B$, Bar graph showing the distribution of branches along axons contacted by FGF- 2 beads. $n$ refers to the number of axon branches found at any part of the axons contacted by beads. Note that branches are located preferentially (57\%) within $10 \mu \mathrm{m}$ of an FGF-2 bead but are distributed randomly along the remaining regions of the axons. $C$, Bar graph comparing the effectiveness of FGF-2 versus BSA beads in promoting branching. Percentages of bead contacts leading to axon branches are calculated for beads only within $10 \mu \mathrm{m}$ of a branch. For BSA beads, $3 \%(n=30)$ were associated with a branch versus $36 \%$ of FGF-2 beads $(n=44)$.

likely to elicit branching if they contacted growth cones versus the axon shaft. Measurements of the distances between the distal tip of the axon and an FGF-2 bead in contact with the axon suggest that the closer the bead was to the growth cone the more likely a branch was to develop. The distance between the primary axonal growth cone and FGF-2-coated beads in contact with the axon was recorded as soon as the beads settled, i.e., $3 \mathrm{hr}$ after the beads were added to the cultures. As shown in Table 1, branches formed near FGF-2-coated beads along the length of the axon. A large proportion of the beads landed on growth cones, perhaps because of the relatively large size of the growth cone compared with the axon shaft. Half of the beads contacting a growth cone elicited branch formation, consistent with results from other studies show- ing sensitivity of the growth cone to branching factors. However, branches also formed near beads that landed on the axon shaft. The percentages of branches that formed within $10 \mu \mathrm{m}$ of a bead that was $>60 \mu \mathrm{m}$ from the growth cone was lower $(\sim 20 \%)$ than when the bead was near the growth cone $(50 \%)$. This tendency suggests that the distal region of the axon including the growth cone is more responsive to branching signals but that branches can also be induced from more proximal regions of the axon shaft. Together, these results suggest that FGF-2 can act on localized regions of the axon to elicit branching and that this branching occurs preferentially in distal regions of the axon close to the growth cone.

\section{DISCUSSION}

In this study, we found that FGF-2 is a potent stimulus for axon branching on cultured cortical neurons that express receptors to FGF-2. Time-lapse imaging over periods of several days showed that bath-applied FGF-2 significantly increased growth cone size and slowed growth cone advance, leading to a threefold increase in axon branching compared with untreated neurons. A previous study of cortical neurons in vitro showed that the terminal growth cone pauses and enlarges in regions from which interstitial axon branches later develop (Szebenyi et al., 1998). Thus, the present results suggest that FGF-2 may increase numbers of axon branches by enhancing the pausing and enlargement of primary growth cones that determine future branch points. Application of FGF-2 was also able to elicit rapid elongation of filopodia within minutes, showing that FGF-2, in addition to long-term effects on branching, also has acute effects on growth cone morphology that may lead eventually to branching. Local release of FGF-2 from heparin beads confirmed that FGF-2 can elicit branches locally by acting on the growth cone or by eliciting branches directly from the axon shaft independent of effects at the primary growth cone. Together, these in vitro results suggest that interstitial axon branches can be induced locally through the action of a targetderived growth factor that preferentially exerts effects on the growth cone but can also act on the axon shaft.

Neurotrophic factors have been shown to regulate aspects of target innervation, particularly the growth of terminal arbors and collateral axon branches (for review, see Henderson, 1996). FGFs participate in many aspects of development (Szebenyi and Fallon, 1999), are widely distributed in the nervous system (Fayein et al., 1992; Eide et al., 1993; Yazaki et al., 1994), and along with their receptors are present in the developing rat cerebral cortex and spinal cord (Riva and Mocchetti, 1991; Weise et al., 1993; Eckenstein, 1994; Kuzis et al., 1995; Vaccarino et al., 1999a). Interestingly, FGF-2 increases significantly in the cortex and spinal cord during the second postnatal week when hamster callosal and corticospinal axons undergo extensive branching in vivo (Norris and Kalil, 1992; Kuang and Kalil, 1994). Receptors to FGF-2 are also present in the embryonic (Raballo et al., 2000) and postnatal (Kuzis et al., 1995) cortex. In a recent study, FGF-2 was found to augment the progenitor pool specific for pyramidal projection neurons (Raballo et al., 2000). Moreover, FGF-2 knock-out mice show abnormal cortical development (Dono et al., 1998; Ortega et al., 1998; Miller et al., 2000), although defects in axon branching were not examined. Thus, FGF-2 in the cortical plate may be especially relevant for the differentiation of pyramidal neurons and, in spinal cord and cortical targets, may influence branching of cortical axons. FGFs enhance neurite branching and process length of hippocampal cells (Miyagawa 
A.

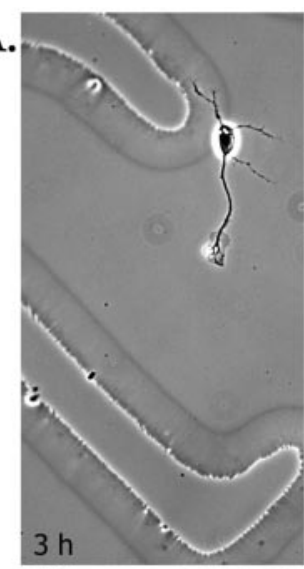

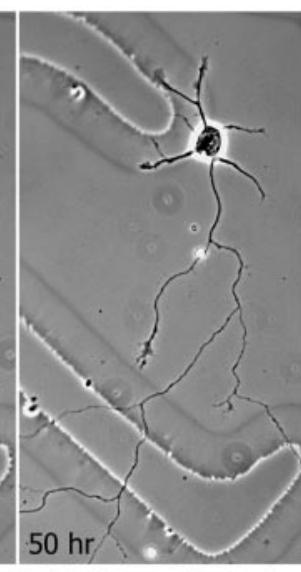

$22 \mathrm{hr}$

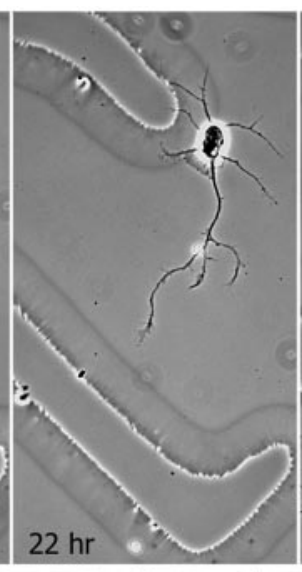

B.

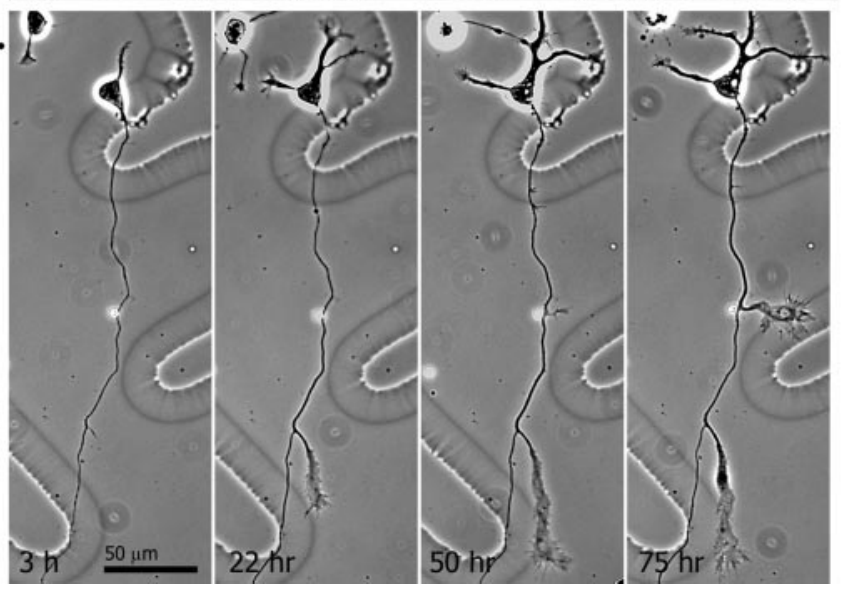

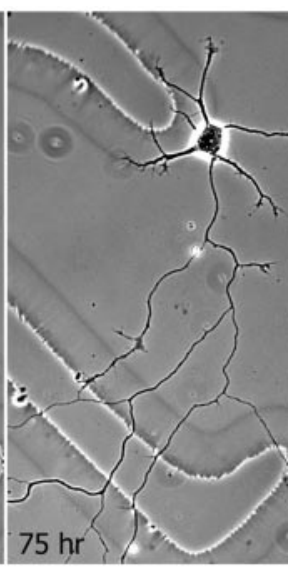

$75 \mathrm{hr}$

Figure 7. FGF-2 beads promote formation of branches from both the growth cone and the axon shaft. $A$, Images of a cortical neuron at increasing time points after an FGF-2-coated bead contacted the axonal growth cone. At $3 \mathrm{hr}$ after bead contact, the growth cone has enlarged, and at $22 \mathrm{hr}$ several branches are emerging near the site of bead contact. These branches continued to grow and rebranched during the following $2 \mathrm{~d}$. $B$, Images of a cortical neuron at increasing time points after an FGF-2-coated bead contacted the axon shaft. A branch tipped by a large growth cone formed on the axon at the point of contact with the bead. Note that another prominent branch also developed spontaneously on the same axon. The bead remained in its original position over time until the last time point ( $75 \mathrm{hr})$.

et al., 1993; Aoyagi et al., 1994; Lowenstein and Arsenault, 1996) and induce sprouting of cholinergic axons in the denervated adult hippocampus (Fagan et al., 1997).

Previous studies have shown that bath or local application of neurotrophic factors can increase protrusive activity on the growth cone and axon shaft within several minutes (Ming et al., 1997). Thin lateral processes emerged from the neurites of frog spinal neurons after lamellipodial activity, but because of the short observation periods it could not be determined whether these processes later developed into mature branches. Our observations showed an increase in filopodial length at the growth cone, suggesting that effects of FGF-2 on these cortical neurons can occur within minutes.

Effects of local application of FGF-2-coated beads to cortical neurons showed that the beads had branch-promoting effects within $10 \mu \mathrm{m}$ of the bead contact and that branches were more

$\begin{aligned} & \text { Table 1. FGF-2 beads promote axon branching preferentially from the } \\
& \text { growth cone }\end{aligned}$
\begin{tabular}{lll}
$\begin{array}{l}\text { Distance from growth } \\
\text { cone to bead }(\mu \mathrm{m})\end{array}$ & $\begin{array}{l}\text { Number of } \\
\text { beads }\end{array}$ & $\begin{array}{l}\text { Number of beads with } \\
\text { a branch within } 10 \mu \mathrm{m}\end{array}$ \\
\hline $0-20$ & 20 & $10(50 \%)$ \\
$20-40$ & 3 & $1(33 \%)$ \\
$40-60$ & 5 & $2(40 \%)$ \\
$>60$ & 16 & $3(20 \%)$
\end{tabular}

The number of branches within $10 \mu \mathrm{m}$ of FGF-2 beads that landed on axons at various distances from the growth cone are shown. The distance from the bead to the growth cone was measured $3 \mathrm{hr}$ after addition of the beads to the culture. The effect of FGF-2 beads on axon branching within $10 \mu \mathrm{m}$ was assessed $3 \mathrm{~d}$ after bead application. likely to form when the bead was in contact with the growth cone rather than more proximal regions of the axon shaft. We also found that FGF-2 from a point source (beads) was far more effective in eliciting branches than bath application. In a recent study of axon collateral sprouting of chick DRG neurons, NGF-coupled beads were shown to induce filopodial sprouts $\sim 20 \mu \mathrm{m}$ long on the axon shaft within 15-20 min of growth factor application (Gallo and Letourneau, 1998). Filopodial activity on DRG neurons required sustained contact with NGF beads for several hours. In contrast, we found that a single FGF-2-coated bead in contact with an axon or a growth cone was able to promote a long (up to $90 \mu \mathrm{m}$ ) stable branch that often rebranched. Branches continued to elongate even when the bead moved away form its original point of contact. The time course for development of axon branches (over $24 \mathrm{hr}$ ) was much longer than for NGF-induced sprouting of filopodia, although filopodia on cortical growth cones also developed within minutes of bath application of FGF-2. Differences in results obtained with growth factor-coated beads suggest that different growth factors may have different modes of action. In contrast to NGF, which is effective when bound to polystyrene beads, FGF-2 must be soluble to have an effect. This is consistent with previous results showing that FGF-2 can be internalized by neurons (Stachowiak et al., 1997; Mufson et al., 1999). Most likely, in vivo, several growth factors work together in concert in the development of collateral branches.

Local and bath application of FGF-2 demonstrate that branches can be stimulated anywhere along the axon shaft. However, we also found that distal regions of the axon, particularly the growth cone, were more responsive to FGF-2 and more likely to extend branches than other regions of the axon shaft. One pos- 
sibility is that distal regions of the axon are more labile because of plasticity in cytoskeletal elements. Newly polymerized more labile microtubules are found preferentially in distal regions of the axon (our unpublished observations; Arregui et al., 1991; Brown et al., 1992). Moreover, direct imaging of microtubules in axons and growth cones has shown that development of axon branches is dependent on local splaying of bundled microtubules and invasion of dynamic microtubule fragments into newly forming branches (Dent et al., 1999). Thus, regions of relative microtubule plasticity may be particularly responsive to FGF-2. Growth factors may also induce cytoskeletal reorganization at branch points, as shown by local debundling of microtubules and high concentrations of actin filaments in regions of filopodial sprouting by DRG axons in contact with NGF-coated beads (Gallo and Letourneau, 1998).

The present study demonstrates that FGF-2 has a profound influence on axon branching. Importantly, we provide novel evidence for the role of growth factors in stimulating interstitial branching through effects on axonal growth cones. Previous studies in situ have shown that growth cones are larger and more complex at decision regions in which they change direction or enter targets. In these regions, growth cones undergo prolonged pausing behaviors (Harris et al., 1987; Kaethner and Stuermer, 1992; Sretavan and Reichardt, 1993; Godement et al., 1994; Mason and Wang, 1997; Skaliora et al., 2000) that often lead to development of interstitial branches that extend into targets (Halloran and Kalil, 1994; Yamamoto et al., 1997). Dissociated cortical neurons in culture also show a dramatic increase in growth cone size during lengthy pausing behaviors (Szebenyi et., 1998; Dent et al., 1999). After the growth cone resumes forward advance, filopodial or lamellipodial remnants on the axon shaft subsequently give rise to axon branches. The present results demonstrate that FGF-2 promotes branching by enhancing these events. Growth cone pausing leading to collateral branching has been observed directly in callosal target regions of living cortical brain slices (Halloran and Kalil, 1994). Moreover, our results have shown that cortical neurons express receptors to FGF-2 at appropriate times in development. Thus, growth cones in vivo may pause and branch at cortical target regions in response to FGF-2, a target-derived factor in the developing cortex (Vaccarino et al., 1999b).

Other experiments in vivo have also suggested a role for FGF-2 in target recognition and axon arborization. Inhibition of FGF receptors in retinal ganglion cells caused their axons to grow more slowly in vitro. When FGF receptors were blocked in vivo, many of the retinal axons failed to innervate their tectal target (McFarlane et al., 1996). Interestingly, some of the axons showed aberrant early branching. These results were interpreted to suggest that normally a decrease in FGF-2 signaling in target regions slows growth cone advance and switches them from a growth mode to an arborizing mode. Thus, retinal axons that do not recognize their target fail to slow down and hence bypass their tectal target. Because FGF-2 levels decrease at the tectal border (McFarlane et al., 1995), retinal growth cones in vivo were thought to detect this decrease in levels of the growth factor by slowing their advance. In contrast, our experiments used bath or local applications of FGF-2 to increase levels of FGF-2. Cortical growth cones responded to these increases by slowing their advance and increasing axon branching. Although the present results and those of McFarlane et al. (1996) might appear to be contradictory, both findings suggest that axons can slow their growth and branch into targets by detecting gradients, i.e., either an increase or a decrease in levels of a target-derived growth factor. In future, it will be important to determine the role of FGF-2 in inducing plastic changes in the axonal and growth cone cytoskeleton (Dent et al., 1999) that underlie development of interstitial axon branches.

\section{REFERENCES}

Aoyagi A, Nishikawa K, Saito H, Abe K (1994) Characterization of basic fibroblast growth factor-mediated acceleration of axonal branching in cultured rat hippocampal neurons. Brain Res 661:117-126.

Arregui C, Busciglio J, Caceres A, Barra HS (1991) Tyrosinated and detyrosinated microtubules in axonal processes of cerebellar macroneurons grown in culture. J Neurosci Res 28:171-181.

Bastmeyer M, O'Leary DD (1996) Dynamics of target recognition by interstitial axon branching along developing cortical axons. J Neurosci 16:1450-1459

Belluardo N, Wu G, Mudo G, Hansson AC, Pettersson R, Fuxe K (1997) Comparative localization of fibroblast growth factor receptor-1, -2 , and -3 mRNAs in the rat brain: in situ hybridization analysis. J Comp Neurol 379:226-246.

Brown A, Slaughter T, Black MM (1992) Newly assembled microtubules are concentrated in the proximal and distal regions of growing axons. J Cell Biol 119:867-882.

Cohen-Cory S, Fraser SE (1995) Effects of brain-derived neurotrophic factor on optic axon branching and remodeling in vivo. Nature 378:192-196.

Delrieu I (2000) The high molecular weight isoforms of basic fibroblast growth factor (FGF-2): an insight into an intracrine mechanism. FEBS Lett 468:6-10.

Dent EW, Callaway JL, Szebenyi G, Baas PW, Kalil K (1999) Reorganization and movement of microtubules in axonal growth cones and developing interstitial branches. J Neurosci 19:8894-8908.

Dono R, Texido G, Dussel R, Ehmke H, Zeller R (1998) Impaired cerebral cortex development and blood pressure regulation in FGF-2deficient mice. EMBO J 17:4213-4225.

Eckenstein FP (1994) Fibroblast growth factors in the nervous system. J Neurobiol 25:1467-1480.

Eide FF, Lowenstein DH, Reichardt LF (1993) Neurotrophins and their receptors: current concepts and implications for neurologic disease. Exp Neurol 121:200-214.

Fagan AM, Suhr ST, Lucidi-Phillipi CA, Peterson DA, Holtzman DM, Gage FH (1997) Endogenous FGF-2 is important for cholinergic sprouting in the denervated hippocampus. J Neurosci 17:2499-2511.

Fayein NA, Courtois Y, Jeanny JC (1992) Basic fibroblast growth factor high and low affinity binding sites in developing mouse brain, hippocampus and cerebellum. Biol Cell 76:1-13.

Gallo G, Letourneau PC (1998) Localized sources of neurotrophins initiate axon collateral sprouting. J Neurosci 18:5403-5414.

Godement P, Wang LC, Mason CA (1994) Retinal axon divergence in the optic chiasm: dynamics of growth cone behavior at the midline. J Neurosci 14:7024-7039.

Grill R, Murai K, Blesch A, Gage FH, Tuszynski MH (1997) Cellular delivery of neurotrophin-3 promotes corticospinal axonal growth and partial functional recovery after spinal cord injury. J Neurosci 17:5560-5572.

Guimond SE, Turnbull JE (1999) Fibroblast growth factor receptor signaling is dictated by specific heparan sulphate saccharides. Curr Biol 9:1343-1346.

Gundersen RW, Barrett JN (1979) Neuronal chemotaxis: chick dorsalroot axons turn toward high concentrations of nerve growth factor. Science 206:1079-1080.

Halloran MC, Kalil K (1994) Dynamic behaviors of growth cones extending in the corpus callosum of living cortical brain slices observed with video microscopy. J Neurosci 14:2161-2177.

Harris WA, Holt CE, Bonhoeffer F (1987) Retinal axons with and without their somata, growing to and arborizing in the tectum of Xenopus embryos: a time-lapse video study of single fibres in vivo. Development 101:123-133.

Henderson CE (1996) Role of neurotrophic factors in neuronal development. Curr Opin Neurobiol 6:64-70.

Itoh N, Yazaki N, Tagashira S, Miyake A, Ozaki K, Minami M, Satoh M, Ohta M, Kawasaki T (1994) Rat FGF receptor-4 mRNA in the brain is expressed preferentially in the medial habenular nucleus. Brain Res Mol Brain Res 21:344-348.

Kaethner RJ, Stuermer CA (1992) Dynamics of terminal arbor formation and target approach of retinotectal axons in living zebrafish embryos: a time-lapse study of single axons. J Neurosci 12:3257-3271.

Kalyani AJ, Mujtaba T, Rao MS (1999) Expression of EGF receptor and FGF receptor isoforms during neuroepithelial stem cell differentiation. J Neurobiol 38:207-224.

Kuang RZ, Kalil K (1994) Development of specificity in corticospinal connections by axon collaterals branching selectively into appropriate spinal targets. J Comp Neurol 344:270-282. 
Kuzis K, Reed S, Cherry NJ, Woodward WR, Eckenstein FP (1995) Developmental time course of acidic and basic fibroblast growth factors' expression in distinct cellular populations of the rat central nervous system. J Comp Neurol 358:142-153.

Letourneau PC (1978) Chemotactic response of nerve fiber elongation to nerve growth factor. Dev Biol 66:183-196.

Lom B, Cohen-Cory S (1999) Brain-derived neurotrophic factor differentially regulates retinal ganglion cell dendritic and axonal arborization in vivo. J Neurosci 19:9928-9938.

Lowenstein DH, Arsenault L (1996) The effects of growth factors on the survival and differentiation of cultured dentate gyrus neurons. J Neurosci 16:1759-1769.

Mason CA, Wang LC (1997) Growth cone form is behavior-specific and, consequently, position-specific along the retinal axon pathway. J Neurosci 17:1086-1100.

McFarlane S, McNeill L, Holt CE (1995) FGF signaling and target recognition in the developing Xenopus visual system. Neuron 15:1017-1028.

McFarlane S, Cornel E, Amaya E, Holt CE (1996) Inhibition of FGF receptor activity in retinal ganglion cell axons causes errors in target recognition. Neuron 17:245-254.

Miller DL, Ortega S, Bashayan O, Basch R, Basilico C (2000) Compensation by fibroblast growth factor 1 (FGF1) does not account for the mild phenotypic defects observed in FGF2 null mice. Mol Cell Biol 20:2260-2268.

Ming G, Lohof AM, Zheng JQ (1997) Acute morphogenic and chemotropic effects of neurotrophins on cultured embryonic Xenopus spinal neurons. J Neurosci 17:7860-7871.

Miyagawa T, Saito H, Nishiyama N (1993) Branching enhancement by basic fibroblast growth factor in cut neurite of hippocampal neurons. Neurosci Lett 153:29-31.

Mufson EJ, Kroin JS, Sendera TJ, Sobreviela T (1999) Distribution and retrograde transport of trophic factors in the central nervous system: functional implications for the treatment of neurodegenerative diseases. Prog Neurobiol 57:451-484.

Niswander L, Tickle C, Vogel A, Booth I, Martin GR (1993) FGF-4 replaces the apical ectodermal ridge and directs outgrowth and patterning of the limb. Cell 75:579-587.

Norris CR, Kalil K (1992) Development of callosal connections in the sensorimotor cortex of the hamster. J Comp Neurol 326:121-132.

O'Leary DD, Terashima T (1988) Cortical axons branch to multiple subcortical targets by interstitial axon budding: implications for target recognition and "waiting periods." Neuron 1:901-910.

O'Leary DD, Bicknese AR, De Carlos JA, Heffner CD, Koester SE, Kutka LJ, Terashima T (1990) Target selection by cortical axons: alternative mechanisms to establish axonal connections in the developing brain. Cold Spring Harb Symp Quant Biol 55:453-468.

Ornitz DM, Xu J, Colvin JS, McEwen DG, MacArthur CA, Coulier F, Gao G, Goldfarb M (1996) Receptor specificity of the fibroblast growth factor family. J Biol Chem 271:15292-15297.

Ortega S, Ittmann M, Tsang SH, Ehrlich M, Basilico C (1998) Neuronal defects and delayed wound healing in mice lacking fibroblast growth factor 2. Proc Natl Acad Sci USA 95:5672-5677.

Raballo R, Rhee J, Lyn-Cook R, Leckman JF, Schwartz ML, Vaccarino FM (2000) Basic fibroblast growth factor (Fgf2) is necessary for cell proliferation and neurogenesis in the developing cerebral cortex. J Neurosci 20:5012-5023.

Riva MA, Mocchetti I (1991) Developmental expression of the basic fibroblast growth factor gene in rat brain. Brain Res Dev Brain Res 62:45-50.

Sawai H, Clarke DB, Kittlerova P, Bray GM, Aguayo AJ (1996) Brainderived neurotrophic factor and neurotrophin- $4 / 5$ stimulate growth of axonal branches from regenerating retinal ganglion cells. J Neurosci 16:3887-3894.

Schnell L, Schneider R, Kolbeck R, Barde YA, Schwab ME (1994) Neurotrophin-3 enhances sprouting of corticospinal tract during development and after adult spinal cord lesion. Nature 367:170-173.

Shitaka Y, Matsuki N, Saito H, Katsuki H (1996) Basic fibroblast growth factor increases functional L-type $\mathrm{Ca}^{2+}$ channels in fetal rat hippocampal neurons: implications for neurite morphogenesis in vitro. J Neurosci 16:6476-6489.

Skaliora I, Adams R, Blakemore C (2000) Morphology and growth patterns of developing thalamocortical axons. J Neurosci 20:3650-3662.

Song HJ, Ming GL, Poo MM (1997) cAMP-induced switching in turning direction of nerve growth cones. Nature 388:275-279.

Sretavan DW, Reichardt LF (1993) Time-lapse video analysis of retinal ganglion cell axon pathfinding at the mammalian optic chiasm: growth cone guidance using intrinsic chiasm cues. Neuron 10:761-777.

Stachowiak MK, Moffett J, Maher P, Tucholski J, Stachowiak EK (1997) Growth factor regulation of cell growth and proliferation in the nervous system. A new intracrine nuclear mechanism. Mol Neurobiol $15: 257-283$

Szebenyi G, Fallon JF (1999) Fibroblast growth factors as multifunctional signaling factors. Int Rev Cytol 185:45-106.

Szebenyi G, Callaway JL, Dent EW, Kalil K (1998) Interstitial branches develop from active regions of the axon demarcated by the primary growth cone during pausing behaviors. J Neurosci 18:7930-7940.

Vaccarino FM, Schwartz ML, Raballo R, Nilsen J, Rhee J, Zhou M, Doetschman T, Coffin JD, Wyland JJ, Hung YT (1999a) Changes in cerebral cortex size are governed by fibroblast growth factor during embryogenesis. Nat Neurosci 2:848.

Vaccarino FM, Schwartz ML, Raballo R, Rhee J, Lyn-Cook R (1999b) Fibroblast growth factor signaling regulates growth and morphogenesis at multiple steps during brain development. Curr Top Dev Biol 46:179-200.

Wang KH, Brose K, Arnott D, Kidd T, Goodman CS, Henzel W, Tessier-Lavigne M (1999) Biochemical purification of a mammalian slit protein as a positive regulator of sensory axon elongation and branching. Cell 96:771-784.

Weise B, Janet T, Grothe C (1993) Localization of bFGF and FGFreceptor in the developing nervous system of the embryonic and newborn rat. J Neurosci Res 34:442-453.

Yamamoto N, Higashi S, Toyama K (1997) Stop and branch behaviors of geniculocortical axons: a time-lapse study in organotypic cocultures. J Neurosci 17:3653-3663.

Yazaki N, Hosoi Y, Kawabata K, Miyake A, Minami M, Satoh M, Ohta M, Kawasaki T, Itoh N (1994) Differential expression patterns of mRNAs for members of the fibroblast growth factor receptor family, FGFR-1-FGFR-4, in rat brain. J Neurosci Res 37:445-452. 